\title{
British Society of Breast Radiology Annual Scientific Meeting 2014
}

\author{
Brighton, UK. 10-11 November 2014
}

Published: 3 November 2014

These abstracts are available online at http://breast-cancer-research.com/supplements/16/S1

\section{ORAL PRESENTATIONS}

01

Multidetector CT Improving Surgical Outcomes in Breast Cancer (MISO BC).

J Cox ${ }^{1 *}$, H Hancock ${ }^{2}$, J Spratt ${ }^{1}$, H Close ${ }^{2}$, CM Lee', U Mohammed ${ }^{2}$, J Mason ${ }^{2}$

${ }^{1}$ County Durham and Darlington NHS Foundation Trust, Durham, UK:;

${ }^{2}$ Durham University, Durham, UK

Breast Cancer Research 2014, 16(Suppl 1):O1

Introduction: This multicentre randomised controlled trial investigated whether a computed tomography (CT) scan of the axilla could more accurately assess whether the axillary lymph nodes were involved with malignancy in patients with newly diagnosed breast cancer and therefore influence surgical decision-making with regard to axillary surgery.

Methods: Patients with newly diagnosed breast cancer (via screening and symptomatic routes) at two NHS Trusts in the North East of England were recruited and randomised in equal numbers. Both groups received routine diagnostic and surgical care (usual care). In addition, one group received a CT scan of their axilla on the same side as the breast cancer.

Results: The study recruited 297 patients, of whom 291 contributed to findings. CT scan-guided care did not result in a change in the need for a second operation, with about $20 \%$ of both groups needing further surgery. Patients within the two groups were similar before treatment, had similar types and grade of cancer, experienced similar pattern complications and reported similar experiences of care.

Conclusion: New diagnostic imaging technologies regularly enter NHS centres of excellence as research tools. It is important these are evaluated rigorously before becoming routine care. In patients newly diagnosed with breast cancer, CT-augmented diagnosis of cancer in the axilla was not found to improve surgical outcomes or patient experience.

\section{2}

Preoperative assessment of breast volume to aid surgical planning: comparison of software-based mammographic measurements with subsequent mastectomy volumes.

I Teo ${ }^{1 *}$, P Whelehan ${ }^{1,2}$, EJ Macaskill ${ }^{1,2}$, D Alex Munnoch ${ }^{1,2}$, S Vinnicombe ${ }^{1,2}$, Andy Evans ${ }^{1,2}$

${ }^{1} \mathrm{NHS}$ Tayside, Dundee, UK.; ${ }^{2}$ University of Dundee, UK.

Breast Cancer Research 2014, 16(Suppl 1):O2

Introduction: The proportion of breast volume excised during conservation surgery for breast cancer is crucial to cosmetic outcomes. Validated, expedient methods for accurate preoperative quantification of breast volume are lacking. This study evaluated breast volume measurements calculated by Volpara ${ }^{\oplus}$ breast density software, by comparing them with actual mastectomy volumes.

Methods: From a prospective clinical database, 31 patients were identified for whom Volpara® (Matakina Technology Limited, New Zealand) volume measurements and mastectomy volumes were available. All patients had undergone skin-sparing mastectomy (SSM), bilateral in one case. Specimen volumes had been measured using a water-displacement technique. Volpara ${ }^{\oplus}$ volumes for the corresponding CC and MLO view of each of the 32 breasts were averaged and compared with the mastectomy volumes. Correlation was assessed using the Pearson correlation coefficient.

Results: Volpara ${ }^{\oplus}$ breast volumes were, as expected, consistently higher than SSM volumes but with a very strong correlation (Pearson correlation coefficient for average Volpara ${ }^{\oplus}$ volumes and mastectomy volumes $=0.82$ $(P<0.01))$.

Conclusion: The excellent correlation between Volpara ${ }^{\circledR}$ and SSM volumes suggests that this readily available and convenient preoperative measure of breast volume could be used as a tool to aid surgical planning in women with breast cancer, which might be particularly useful in those women not scheduled for preoperative MRI. When coupled with accurate measurement of tumour size plus required excision margin volume, possibly from three-dimensional ultrasound, this tool could potentially outperform current subjective preoperative methods of assessing relative volumes. Further validation work is needed.

\section{3}

Efficacy of second-time vacuum-assisted breast excision in the management of screen-detected high-risk breast lesions.

M Morgan ${ }^{1,2^{*}}$, R Patel $^{1,2}$, W Teh $^{1,2}$

${ }^{1}$ Northwick Park Hospital, Harrow, UK.; ${ }^{2}$ North London Breast Screening,

London, UK.

Breast Cancer Research 2014, 16(Suppl 1):O3

Introduction: Surgical excision of breast lesions is usually required for high-risk (B3) lesions or radiological pathological discordance. The risk of underestimation of malignancy is 45 to $50 \%$ for $14 \mathrm{G}$ core biopsy and 20 to $25 \%$ for vacuum-assisted biopsies (VAB).

Methods: In our institution, all screen-detected microcalcifications or ultrasound occult lesions are subjected to $11 \mathrm{G} \mathrm{VAB}$. If a discordant or high-risk lesion (B3) - for example, atypical ductal hyperplasia (ADH), lobular neoplasia or papillary lesion - is diagnosed on the initial VAB, an $8 \mathrm{G}$ vacuum-assisted breast excision (VAE) may be performed following MDT discussion.

Results: Between 2011 and 2013, 1,250 VAB were performed on 990 women. Eighty-three $8 \mathrm{G}$ VAEs were performed during this time, and of these $62.7 \%$ of lesions $(52 / 83)$ were classified histologically as B2, $24.1 \%$ (20/83) remained $\mathrm{B} 3,2.4 \%(2 / 83)$ as $\mathrm{B} 4,7.2 \%(6 / 83)$ as $\mathrm{B} 5$ a and $2.4 \%(2 / 83)$ as B5b. Following $8 \mathrm{G} \mathrm{VAE,} 45$ patients $(54.2 \%)$ were discharged to screening, six patients $(7.2 \%)$ underwent annual surveillance, diagnostic surgery was performed on 23 patients $(27.7 \%)$ and therapeutic surgery on eight patients (9.6\%).

Conclusion: Our study demonstrates that $8 \mathrm{G}$ VAE after an initial B3 diagnosis on $V A B$ can avoid diagnostic surgery and improve cancer detection. Surgery was not required in 51 of the 83 cases $(61.4 \%)$ of B3 
lesions found on initial 11G VAB. A total of 17 B3 lesions (20.5\%) were upgraded after $8 \mathrm{G} V A E$; nine by diagnostic surgery $(12 \%)$ and eight of the 83 cases of $\mathrm{B} 3$ lesions (9.6\%) by VAE to either $\mathrm{B} 5 \mathrm{a}$ or $\mathrm{B} 5 \mathrm{~b}$ requiring definitive surgery. Lesions most likely to be upgraded to malignancy were lobular neoplasia and ADH.

\section{4}

Local breast density at lesion sites in diagnostic and previous screening mammograms.

M Otsuka ${ }^{1 *}$, E Harkness $^{2,3}, X$ Chen $^{2}$, E Moschidis $^{2}$, M Bydder $^{3}$, S Gadde $^{3}$, Y Lim ${ }^{2,3}$, A Maxwell ${ }^{2,3}$, DG Evans ${ }^{3,4}$, A Howell ${ }^{3,4}$, P Stavrinos ${ }^{3,5}$, M Wilson ${ }^{3}$, S Astley ${ }^{2,4}$

${ }^{1}$ University of Manchester Medical School, Manchester, UK: ${ }^{2}$ Centre for Imaging Sciences, Institute of Population Health, University of Manchester, UK; ${ }^{3}$ Nightingale Centre and Genesis Prevention Centre, University Hospital of South Manchester, Manchester, UK:; ${ }^{4}$ Manchester Breast Centre, Manchester Cancer Research Centre, University of Manchester, UK.; ${ }^{5}$ The University of Manchester, Manchester Academic Health Science Centre, University Hospital of South Manchester NHS Foundation Trust, Manchester, UK.

Breast Cancer Research 2014, 16(Suppl 1):04

Introduction: High overall breast density is associated with increased risk of developing cancer. Semi-automated analysis of digitised analogue mammograms has previously suggested that local increases in density may occur prior to cancers being detected. We investigate local density at the site of cancers in diagnostic and previous full-field digital screening mammograms using quantitative measures.

Methods: Volpara ${ }^{\circledR}$ volumetric breast density maps were obtained for 54 mammograms in which unilateral breast cancer was detected, and the corresponding previous digital screening mammograms that had been read as normal. A $5 \mathrm{~mm}$ square region was sampled from CC-view density maps at the lesion site in both diagnostic and previous screening mammograms, and in corresponding locations on the opposite side. Local percent density was computed.

Results: In previous screening mammograms, local breast density was significantly increased at the future lesion site compared with a similar location in the opposite breast (medians $18.82 \%, 9.45 \%, P<0.001$ ). Breast density at the lesion site in diagnostic mammograms was higher than that of a corresponding area in the opposite breast (medians $21.58 \%$, $9.18 \%, P<0.001)$. It was also greater than the density of the same location in the previous screening mammogram $(P=0.012)$.

Conclusion: Local breast density is increased at sites where cancer will develop in the future compared with corresponding regions in the opposite breast. Cancer sites in diagnostic images have greater density than the same region in previous screening mammograms and corresponding contralateral regions. Detection of localised increases in breast density could enhance computer-aided detection of early breast cancer.

\section{5}

Are abnormalities missed in the PERFORMS self-assessment scheme due to visual or cognitive factors?

Y Chen", L Dong, A Gale

Loughborough University, Loughborough, UK.

Breast Cancer Research 2014, 16(Suppl 1):05

Introduction: To examine whether missed abnormalities in PERFORMS test sets are mainly due to visual or cognitive factors.

Methods: Data were examined from three recent rounds of the PERFORMS breast screening self-assessment scheme (that is, three sets of 120 difficult normal, benign and malignant FFDM cases) that had respectively been reported by 723,726 and 687 breast radiologists and advanced practitioners in order to determine the error rates and the underlying reasons for such errors. Every participant had read each case set in a different random order. Results: The cancer detection in the three rounds was high, with mean values of $82 \%, 86 \%$ and $93 \%$. However, in terms of discrepant recall decisions between individuals and overall decisions, there were both overreading (for the normal and benign cases) and under-reading errors (for the malignant cases) for many cases; although there was no statistical difference in the error rate between these three case categories. Overall, for the normal cases there were $15.55 \%$ false positives, with the majority of these being scored as indeterminate (13.37\%). For malignant cases there were $16 \%$ false negatives, with $11.45 \%$ reported normal and $4.12 \%$ benign. Further analyses demonstrated that the majority of the false negative errors were due to mammographic features being missed (unseen) rather than being seen but then misinterpreted - a cognitive error ( $t$ test, $P<0.002)$.

Conclusion: In examining these difficult case sets from the PERFORMS scheme, most errors were due to key abnormal mammographic features not being seen.

06

Digital breast tomosynthesis improves the accuracy of the diagnosis of circumscribed lesions because of increase of margin visibility.

R Wasan*, J Morel, A Iqbal, D Evans, J Goligher, C Peacock, R Rahim,

K Satchithananda, M Michell

King's College Hospital, London, UK.

Breast Cancer Research 2014, 16(Suppl 1):O6

Introduction: Circumscribed masses are a common cause of recall for assessment but most are benign. The aim of this study is to compare the accuracy of digital breast tomosynthesis (DBT) with digital mammography (DM) in assessing the margin of circumscribed lesions as a predictor of benign or malignant disease.

Methods: The study group consisted of women recalled from breast screening for further assessment of circumscribed masses. Clients underwent co-registered DM and DBT in both MLO and CC projections. Two experienced breast radiologists evaluated DM and DBT images and a consensus decision was reached on the percentage of the margin that was well defined on DM and DBT. The lesions were categorised 1 to 4 as follows: $1=0$ to $25 \%, 2=26$ to $50 \%, 3=51$ to $75 \%$ and $4=76$ to $100 \%$.

Results: One hundred and twenty circumscribed lesions were evaluated. Data on 118 lesions seen on the MLO view are presented. There were 93 benign lesions and 25 cancers. There was a change in distribution of margin categories between DM and DBT. More lesions were categorised as 3 or 4 on DBT (59/118) compared with DM (18/118). Of the 93 benign lesions, 17 were categorised as 3 or 4 on DM and 57 on DBT. The difference between the two proportions was significant $(P<0.0002)$. There were more cancers categorised as 1 on DBT $(21 / 39=54 \%)$ compared with DM $(20 / 76=26 \%)$; Fisher's exact test $(P<0.004)$.

Conclusion: Increased margin visibility of circumscribed masses by DBT improves the accuracy of mammography interpretation and may decrease the recall rate in mammography screening.

\section{POSTER PRESENTATIONS}

\section{P1}

PB.34. Marker clip placement may not be needed in breast cancer patients undergoing neoadjuvant chemotherapy with a view to breast-conserving surgery whose tumours are associated with microcalcification.

M Sreenivas

UHCW Hospitals NHS Trust, Coventry, UK

Breast Cancer Research 2014, 16(Suppl 1):P1

Introduction: Patients who are potential candidates for breast conservation surgery following neoadjuvant chemotherapy have their tumours marked with a maker clip to guide surgery. Some of the breast cancers are associated with microcalcification. So far we have not seen any publication showing the potential use of tumour-associated microcalcification, which could be used as marker of the tumour bed instead of a marker clip. We present prospective case series where microcalcification associated with tumour had been used to guide surgery.

Methods: Thirty-eight patients with breast cancers underwent neoadjuvant chemotherapy between 1 December 2012 and 7 May 2014 at a single institute. At the end of chemotherapy 16 patients underwent successful breast-conserving surgery (BCS). Nineteen patients' tumours were associated with microcalcification.

Results: Of the patients who underwent successful BCS, seven had tumourassociated microcalcification. In six of these patients the tumour-associated microcalcification was solely used as a marker of the tumour bed. In one case, due to the presence of few tiny eccentric specks of microcalcification 
associated with the tumour, it was decided to place a maker clip. In all seven cases, tumour-associated microcalcification was readily apparent during localisation.

Conclusion: Traditionally, placement of commercially available markers clips (costs around $£ 55$ per clip) is recommended for patients considered suitable for BCS following neoadjuvant chemotherapy. Albeit a small series, our experience demonstrates that tumour-associated microcalcification will still be apparent after completion of neoadjuvant chemotherapy and as such can be used as a marker of the tumour bed, thus saving costs associated with clip placement.

\section{P3}

PB.12. Audit and root-cause analysis of classification 2 and 3 interval cancers.

D Cox*, A Powell

Sandwell \& West Birmingham Hospitals NHS Trust, Birmingham, UK. Breast Cancer Research 2014, 16(Suppl 1):P3

Introduction: The definition of an interval cancer is a cancer diagnosed between scheduled screening rounds. Interval cancer rates are an indication of the effectiveness of a Breast Screening Service in achieving early cancer detection, thus reducing overall mortality secondary to breast cancer, and represent a National Health Service Breast Screening Programme (NHSBSP) standard. The British Society of Breast Radiology (BSBR) Interval Classifications are as follows: $0=$ unclassifiable, $1=$ normal $/$ benign, 2 = uncertain and $3=$ suspicious. An audit was undertaken of interval cancers assigned a classification of 2 or 3 within our Breast Service over a 3-year period with the aim of determining and implementing improved clinical practice in a learning environment.

Methods: The screening and symptomatic records were collected for al NHSBSP clients over a 3-year period subsequently diagnosed with a classification 2 or 3 interval cancer to establish the interval cancer rate benchmarked against NHSBSP standards and to determine the causal factors within the screening pathway.

Results: During the audit period 93,296 women underwent breast screening. The total number of interval cancers was 220 . Of these 52 were assigned a 2 or 3 interval cancer classification. The NHSBSP standard of 1.2 interval cancers per 1000 women screened for the first 2 years following screening was not achieved for women screened within the initial 2 years of the audit period.

Conclusion: Causal factors were most commonly associated with double mammographic screen reading. Subsequent interval cancers occurring most commonly during an incident screen in women aged 50 to 59 years, 12 to 24 months following NHSBSP screening.

\section{P4}

PB.6. Management of radial scars on core biopsy.

L Hodkin*, R Millican-Slater, N Sharma

Leeds Teaching Hospitals Trust, Leeds, UK.

Breast Cancer Research 2014, 16(Suppl 1):P4

Introduction: Radial scar (RS) is a pathological diagnosis which is classified as B3 indeterminate due to the associated risk of malignancy. Further tissue sampling is therefore required, which traditionally was with surgical excision. In Leeds since 2009 we have been managing RS with vacuum-assisted biopsy, if $<15 \mathrm{~mm}$ with no atypia; otherwise surgical management is taken. We reviewed cases since 2009 to identify how RS has been managed and if this is appropriate.

Methods: From 1 April 2009 to 31 March 2013 all RSs assigned B3 on histology were identified. The imaging features, size, histology and subsequent management were documented.

Results: Sixty-four patients were identified, 48 from breast screening and 16 symptomatic. Size of RS ranged from 2 to $70 \mathrm{~mm}$. On imaging, five presented as asymmetry, one as asymmetry with calcification, 40 stromal deformities, five stromal deformities with calcification, four masses, eight calcifications and one normal. Overall, $13 \mathrm{RSs}$ had associated atypia (three cancers) and 51 with no atypia on biopsy had six associated cancers on diagnostic surgery (RS $>20 \mathrm{~mm}$ ) - three LCIS and three DCIS. In total, 27/64 had diagnostic surgical biopsy (five had VAB preoperatively) and 37 had second-line $\mathrm{VAB}$, of which seven are on 5-year FU.
Conclusion: Yield of malignancy for small $(<20 \mathrm{~mm})$ RS with no atypia is low, therefore management with second-line $V A B$ is appropriate rather than surgical diagnostic biopsy, providing there is radiological and pathological correlation with representative sampling.

RS with atypia still requires diagnostic excision, with a $23 \%$ upgrade to cancer discovered from our cases.

P6

PB.13. Impact on film reading of moving from analogue to digital screening: a service evaluation - South East Scotland Breast Screening Programme.

G Babu*, A Gilchrist, J Murray

NHS Lothian, Edinburgh, UK.

Breast Cancer Research 2014, 16(Suppl 1):P6

Introduction: In 2013 this unit became the first in Scotland to switch from analogue to digital mammographic screening. This study analyses the impact of this change on film reading practice with a reflective questionnaire and comparative analysis of recall, arbitration rates and positive predictive values (PPV).

Methods: Film readers in this unit are radiologists, radiographers and clinicians. A questionnaire was designed to assess individual reading practice before and after the switch to digital screening including perception of lesion types. We used SBSP reports and internal statistics for the comparative analysis.

Results and conclusion: Eleven of 13 film readers responded. All readers agreed they enjoyed digital reading. All readers agreed the ability to manipulate images was useful. Most readers felt they would have liked more training when digital mammography was introduced. Eight readers felt digital was convenient and easier to read. Three felt it took longer. Several recorded intermittent eye strain. Readers found comparison with previous analogue films problematic. All readers review their reading figures annually. Nonradiologists do formalised self-audit, which continues after training. This tool is less used by radiologists, who obtain informal feedback from clinics and MDM. Fifty per cent of readers felt that reading guidelines would be helpful. With digital reading, all were confident reading opacities but recalling more calcifications. Readers were generally less confident with asymmetries and parenchymal distortions. Using two comparable 6-month periods, recall rates showed an initial peaking with introduction of digital screening. Arbitration rates increased but PPV significantly improved.

P7

PB.14. An analysis of subtle alterations in reading pattern with the introduction of digital mammography screening: South East Scotland Breast Screening Programme.

G Babu*, A Gilchrist, J Murray

NHS Lothian, Edinburgh, UK.

Breast Cancer Research 2014, 16(Suppl 1):P7

Introduction: Digital screening was implemented in this programme in November 2013. Following an impression that the pattern of recalls had changed, this study aimed to identify cases recalled for subtle or vague mammographic features which were true lesions and those where there was overcall, and perhaps suggest guidance for readers.

Methods: A total of 235 recall cases were randomly identified between November 2013 and March 2014. All were possible subtle lesions, parenchymal deformity or asymmetry. Definite opacities, calcifications and symptoms were excluded. The cases were reviewed by two experienced film readers and a specialist trainee and categorised as Category 1, definite overcall; Category 2, Possible overcall; category 3, definite recall.

Results and conclusion:

Following assessment, all cases in Category 1 were composite norma breast tissue (NBT) and returned to normal routine recall. In Category 2, 37 were composite NBT; 19 were real lesions, mostly cysts; four were cancers. In Category 3, 51 were real lesions, mostly cysts; 17 were cancers. This study has demonstrated that in Category 1 cases (definite overcall), no cancers would have been missed. In Category 2 cases (possible overcall), we must accept some overcall as this group can hide subtle cancers. In Category 3 cases (definite recall), almost all warranted recall. The cancers 
appeared suspicious on screening mammograms. The difference in feature perception between digital and analogue mammography is significant and may alter recall practice. Critical analysis of subtle appearances is required to prevent overcall for future readers.

P8

PB.41. What is the incidence of internal mammary node lymphadenopathy on CT in primary breast cancer patients within 1 year of a diagnosis?.

S Savaridas ${ }^{*}$, J Cox

University Hospital of North Durham, Durham, UK.

Breast Cancer Research 2014, 16(Suppl 1):P8

Introduction: The importance of internal mammary nodes (IMNs) in the staging and treatment of breast cancer patients is controversial. Lymphoscintigraphic studies have demonstrated that a significant proportion of breast cancers have primary or partial IMN drainage. However, historical studies demonstrated no overall benefit of treating IMNs with either surgery or radiotherapy. But with recent advances in treatment, targeted radiotherapy of the IMN chain is now possible. Thus we sought to establish the frequency of IMNs within our primary breast cancer population. Methods: Retrospective cohort study of all spiral CT thorax performed on patients within 12 months following the primary diagnosis of breast cancer from January 2009 to December 2012. The number and size of any IMNs were recorded.

Results: A total of 830 patients were diagnosed with primary breast cance within our time frame; 150 patients were included in this study. Of these, $40 \%(n=60)$ had IMNs present, although the majority were small $(<5 \mathrm{~mm})$. However, $16 \%(n=24)$ had larger nodes $>5 \mathrm{~mm}$ present on CT.

Conclusion: We have demonstrated that IMNs are present in a substantial number of our primary breast cancer patients. We suggest that routine imaging of the IMN chain as well as axilla should be considered in the staging of breast cancer.

\section{P9}

PB.42. Influences upon presentation to the symptomatic breast clinic: is there an increase in symptomatic referrals due to the physical presence of the breast screening van within an area?.

F Wotton ${ }^{1 *}$, R Green

'Peninsula Radiology Academy, Plymouth, UK.; ${ }^{2}$ South Devon Healthcare NHS Foundation Trust, Torquay, UK.

Breast Cancer Research 2014, 16(Suppl 1):P9

Introduction: Current published literature on health-seeking behaviour in women with a self-detected breast lesion mainly explores the reasons surrounding why a delayed presentation may occur. However, there is no current literature on whether the presence of the National Breast Screening van has any influence on symptomatic referrals. Therefore, the authors propose there will be an increase in symptomatic referrals within the cohort of patients who share a postcode with the breast screening van location.

Methods: The National Breast Screening van was parked in the TQ4 postcode between 30 November 2012 and 30 June 2013. All new GP symptomatic breast referrals between the above dates and those during the same timeframe of the previous year were recorded by age and postcode. The referral patterns, ages and number of new symptomatic breast cancer diagnoses between were compared between the study timeframes.

Results: There was a $12 \%$ increase in overall referrals during the study timeframes in 2011/12 to 2012/13. There were no significant differences in referral patterns by postcode. However, there was a statistically significant increase in referrals in younger patients with a consequent reduction in those of screening age $(P<0.001)$, when compared with a separate postcode. No difference in new symptomatic breast cancer diagnoses was seen during the two timeframes.

Conclusion: Whilst the presence of the screening van did not influence overall referrals, there was a shift in age distribution with a statistically significant increase in younger, prescreening women and a consequent decrease in older, screening-age women. The screening van did not influence the number of new symptomatic breast cancers.
P10

PB.26. MRI-guided breast biopsy in Leeds: 12 years' experience.

S Rajan *, N Sharma, B Dall

Breast Imaging Unit, St James's University Hospital Trust, Leeds, UK.

Breast Cancer Research 2014, 16(Suppl 1):P10

Introduction: MRI-guided biopsy allows sampling of lesions occult on conventional imaging to reach a preoperative histological diagnosis and guide patient management.

Methods: We performed a retrospective audit of all MRI-guided biopsies performed in Leeds patients over a 12-year study period from the initiation of this service in April 2002. Data collected: indication for MRI; pre-biopsy MRI score; histology from MRI-guided biopsy and final surgery.

Results: There were $46 \mathrm{MR}$-guided breast biopsies performed (symptomatic $n=26$, screening $n=20$ ). Indication for MRI was to assess extent of malignancy $(n=29)$, an adjunct to triple assessment $(n=11)$ and a screening investigation in higher risk patients $(n=6)$. Pre-biopsy MRI score was classified as indeterminate MRI $3(n=22)$, suspicious MRI $4(n=20)$ and malignant MRI $5(n=4)$. In the MRI 3 subgroup, five proved malignant at MR-guided biopsy with a positive predictive value of $23 \%(5 / 22)$. In the MRI 4/5 subgroup, 14 proved malignant at MR-guided biopsy and a further four malignant lesions were identified at diagnostic surgical biopsy, with an overall positive predictive value of $75 \%(18 / 24)$.

Conclusion: MRI-detected lesions are interpreted in clinical context and attributed an MRI score. If the patient has an increased risk of cancer or there already is a cancer present, any additional lesion should be regarded with a higher level of suspicion. MRI 4/5 lesions have a high positive predictive value for malignancy and should proceed to diagnostic surgical biopsy if the initial MR-guided biopsy is inconclusive.

P15

PB.22. Does mammographic compression force at breast screening influence the likelihood of subsequent screening attendance?.

J Meyer ${ }^{1 *}$, A Maxwell $^{2,3}$, E Harkness $^{2,3}$, S Astley ${ }^{2,3}$, C Mercer $^{3}$, M Wilson $^{3}$, M Bydder', Y Lim $^{3}$, J Morris $^{4}$

${ }^{1}$ University of Manchester Medical School, Manchester, UK.; ${ }^{2}$ Centre for Imaging Sciences, Institute of Population Health, University of Manchester, UK: ${ }^{3}$ Nightingale Centre and Genesis Prevention Centre, University Hospital of South Manchester, Manchester, UK.; ${ }^{4}$ University Hospital of South Manchester, Manchester, UK.

Breast Cancer Research 2014, 16(Suppl 1):P15

Introduction: Previous work has demonstrated that approximately $14 \%$ of women who attend their first breast screening appointment fail to attend the next appointment. It is well recognised that some women experience marked discomfort or pain during mammography which may be related to the breast compression force applied. This study investigates whether applied compression force is related to the likelihood of attendance for the next scheduled screening mammogram.

Methods: A search on the national breast screening database identified women aged 46 to 53 who attended for prevalent screening in 2009/10 and their attendance status at the next invited screen was recorded. A total of 238 subsequent nonattenders were identified together with a sample of 240 women who had subsequently attended that had a similar age distribution. The compression force used for each of the prevalent screening images for these 478 women was recorded.

Results: The median age of the women in both groups was 50 years. The mean compression force applied for all images during the prevalent screen was $102.1 \mathrm{~N}$ for subsequent attenders and $104.4 \mathrm{~N}$ for subsequent nonattenders $(P=0.263)$. There were similarly no significant differences between the two groups for maximum compression force $(P=0.410)$ or compression force for individual views (LMLO, $P=0.200$; RMLO, $P=0.605$; $\mathrm{LCC}, P=0.903 ; \mathrm{RCC}, P=0.246$ )

Conclusion: No statistically significant relationship has been established between mammographic compression force and the likelihood of subsequent screening attendance. Other factors such as compression pressure (compression force per unit area of contact) may be more important, and further research is planned to investigate this. 
P16

PB.1. Axillary lymph node ultrasound and needle sampling in preoperative staging of breast cancer: re-audit.

P Hamilton ${ }^{1 *}, \mathrm{H} \mathrm{Kazi}^{2}$, L Clarke ${ }^{1}$, A Robinson' ${ }^{1}$, A Leaver ${ }^{1}$

'Gateshead Hospitals NHS Trust, Gateshead, UK.; ${ }^{2}$ South Tyneside Hospitals NHS Trust, South Tyneside, UK.

Breast Cancer Research 2014, 16(Suppl 1):P16

Introduction: In our unit, invasive breast cancer patients undergo axillary ultrasound and sometimes needle sampling with fine-needle aspiration or core biopsy for preoperative staging. Results triage patients to appropriate axillary surgery. We present a complete audit cycle. Between cycles 1 and 2, departmental guidelines were changed to include repeat preoperative biopsy in cases with suspicious ultrasound but negative biopsy result (LN4 and LN5 of an LN1 to LN5 node ultrasound grading system), and for patients with inconclusive biopsy results.

Methods: The authors performed a retrospective analysis of multidisciplinary team meeting records of all invasive breast cancer patients operated upon from January 2010 to June 2010 (cycle 1), and from January 2012 to December 2013 (cycle 2). Descriptive statistics were performed.

Results: Initial audit (cycle 1): 125 female patients with 64\% (921/33) overall combined sensitivity of ultrasound/needle biopsy and $100 \%$ (92/92) specificity. Re-audit (cycle 2): 676 female patients, axillary ultrasound performed in all cases. Needle sample performed in $52.8 \%$ (357/676) patients. Repeat needle sampling in 16\% (57/357) patients: $77.2 \%$ (44/57) benign, 22.8\% (13/57) malignant. Ultrasound sensitivity $79.1 \%(163 / 206)$, needle sampling sensitivity $76.1 \%$ (124/163). Overall combined sensitivity of ultrasound/needle sampling 59\% (466/469). Overall combined specificity of ultrasound/needle sampling was $99.3 \%$ (466/469).

Conclusion: The introduction of repeat preoperative lymph node biopsy guidelines did demonstrate node metastases that were not seen on initial node biopsy, increasing accuracy of triage to an appropriate axillary surgical procedure in those patients. Unfortunately this did not lead to an improvement in overall sensitivity of preoperative axillary staging between cycles.

\section{P17}

PB.27. Breast screening with MRI in high-risk women.

D Howarth*, J Gilmour, N Sibal, CE Holmes, N Forester, L McLean

Breast Screening and Assessment Unit, Newcastle, UK.

Breast Cancer Research 2014, 16(Suppl 1):P17

Introduction: This study evaluates the use of annual breast MRI surveillance for high-risk women, evaluating the recall rate for further imaging, biopsy and cancer detection rate. We have used breast MRI for surveillance of highrisk women since 2008.

Methods: High-risk women identified by the Regional Genetic Service between 2008 and 2013 underwent annual breast MRI (1.5 T), in some cases in conjunction with digital mammography. This included those with a genetic predisposition of breast cancer (TP53, BRCA-1, BRCA-2) or equivalent high risk and previous thoracic radiotherapy. Women were offered MRI screening between the ages of 20 and 50 .

Results: Over 6 years, 184 women underwent 477 screening MRIs. There were 44 lesions recalled for second-look ultrasound in 39 women. Twentyseven lesions had a core biopsy, from which 10 malignant lesions were identified. Nine of these were invasive ductal carcinoma, of which all were either grade 2 or 3 , varying in size from 4 to $34 \mathrm{~mm}$. One case of ductal carcinoma in situ was identified. The remaining 17 lesions were benign. Women who had a normal ultrasound either had an interval MRI after 6 months or were returned to routine yearly screen.

Conclusions: MRI screening of high-risk women is effective, with a cancer detection rate of $2.1 \%$ and a recall rate of $9.2 \%$ which are within the standards set by UK national guidelines. There has been one false negative screen to date. Breast MRI screening provides a viable alternative to prophylactic mastectomy for women at high risk of breast cancer.
P18

PB.2. Axillary node ultrasound in breast cancer: which threshold for

'diffuse cortical thickening' predicts node metastasis?.

P Hamilton*, L Clarke, A Leaver

Gateshead Hospitals NHS Trust, Gateshead, UK.

Breast Cancer Research 2014, 16(Suppl 1):P18

Introduction: There is variation in the literature and between breast units in the diffuse cortical thickening (DCT) threshold used for preoperative lymph node needle sampling. In our centre, needle sampling is performed in patients with DCT of $2 \mathrm{~mm}$ and above (LN3 of an LN1 to LN5 ultrasound classification). Results triage patients to appropriate axillary surgery: sentinel node biopsy or axillary clearance. This study investigates our $2 \mathrm{~mm}$ needle sampling threshold, to determine whether we can safely increase to 2.3 or $3 \mathrm{~mm}$ as used in some centres.

Methods: The authors performed a retrospective audit reviewing multidisciplinary team meeting records and ultrasound images of all invasive breast cancer patients with LN3 nodes diagnosed and operated upon in 2012 and 2013. Positive predictive value (PPV) was calculated for DCT ranges and a post-test probability of a malignant needle sample, and then a surgical yield of greater than two metastatic nodes.

Results: A total of 223 female patients had an LN3 result and underwent needle sampling. The PPV for a malignant needle sample for DCT in the ranges 2.0 to $2.29 \mathrm{~mm}, 2.3$ to $2.99 \mathrm{~mm}$ and $\geq 3.0 \mathrm{~mm}$ were $4.1 \%(1 / 24), 10.8 \%(12 / 111)$ and $17.4 \%(15 / 86)$. The PPV for a surgical yield of greater than two metastatic nodes in the ranges 2.0 to 2.29 $\mathrm{mm}, 2.3$ to $2.99 \mathrm{~mm}$ and $\geq 3.0 \mathrm{~mm}$ were $16.6 \%(4 / 24), 4.5 \%(5 / 111)$ and $9.3 \%(8 / 86)$.

Conclusion: The $2 \mathrm{~mm}$ threshold leads to more accurate patient triage to appropriate axillary surgery, but requires many patients to undergo needle sampling for a small positive yield. Even the $2 \mathrm{~mm}$ DCT threshold does not identify all patients with bulky ( $>2$ nodes) metastatic disease.

P19

PB.35. How does semi-automated computer-derived CT measure of breast density compare with subjective assessments to measure mean glandular breast density in patients with breast cancer?.

G Bansal*, S Yapa

University Hospital Llandough, Penarth, UK.

Breast Cancer Research 2014, 16(Suppl 1):P19

Introduction: The objectives were to compare radiologists' breast mammographic density readings with computed tomography (CT) subjective measures; and to correlate computer-derived measurement of $\mathrm{CT}$ density with subjective assessments.

Methods: Retrospective review of mammograms and CT scans in 77 breast cancer patients obtained within 1 year of each other was performed. Two radiologists independently reviewed both $\mathrm{CT}$ and mammograms and classified each case into four categories as defined by the Breast Imaging, Reporting and Data system of the American College of Radiology. Interreader agreements were obtained for both mammographic and CT density subjective evaluations by using the Cohen weighted $k$ statistic. Correlation was also sought between subjective $\mathrm{CT}$ density measurement and mammographic density measurements for each reader using Spearman correlation coefficient. The semi-automated computer-derived measurement of breast density was correlated with visual measurements.

Results: Inter-reader agreements was lower for subjective CT density grades than for mammographic readings, $0.428(\mathrm{Cl}: 0.24$ to 0.89$)$ versus 0.571 (Cl 0.35 to 0.76$)$, respectively. There was moderately good correlation between subjective CT density grades and the mammographic density grades for both readers $(0.760$, Reader $1 ; 0.913$, Reader 2$)$. The semiautomated CT density measurement correlated well with the subjective assessments, with complete agreement of the density grades in $84.9 \%$ of patients and only one-level difference in the rest.

Conclusions: Semi-automated CT density measurements in the evaluation of breast density correlated well with subjective mammographic density measurement. Further studies are needed to incorporate this extra information from a CT scan in the risk stratification of patients. 
P20

PB.7. Large-volume biopsy for B3 lesions without atypia: is once enough?.

N Forester

Breast Screening and Assessment Unit, Newcastle, UK.

Breast Cancer Research 2014, 16(Suppl 1):P20

Introduction: B3 lesion incidence has increased with digital mammography and screening of younger women. In January 2012 we changed management of B3 lesions, following pathways published by Rajan and colleagues. This study reviews the outcome of vacuum-assisted biopsy (VAB) in all patients with a B3 diagnosis, comparing initial core and subsequent $\mathrm{VAB}$ for concordance.

Methods: A single-centre, prospective study of all B3 lesions from January 2012 to June 2014 diagnosed by 14G (US) or 7 to 10G (stereotactic) biopsy with subsequent 7 to $8 \mathrm{G} \mathrm{VAB}$.

Results: Over 30 months, 258 B3 lesions were identified in 255 women. Second-line VAB was performed for 192 lesions (66 no further intervention/excision biopsy at pathologist request). In total, 79/192 were lesions without atypia (fibroepithelial/papillary/RS/CSL) from which three were upgraded by VAB (two B5a, one B4). All of the upgraded B3 lesions without atypia had an initial 14G core. In total, 113/192 were lesions with atypia (ADH/AIDP/FEA/LISN/LCIS/papillary/RS/CSL) from which 21 were upgraded by VAB (18\%). VAB and initial histology were concordant in $87 \%$ of lesions.

Conclusion: VAB in $B 3$ lesions with atypia has a significant and important upgrade rate to malignancy. However, in the absence of atypia the upgrade rate is very low $(<4 \%)$. In this group, there was no discordant pathology between an initial $10 \mathrm{G}$ stereotactic core and subsequent 7 to $8 \mathrm{G}$ large-volume biopsy. To reduce unnecessary intervention and overdiagnosis, we would like to propose that one $10 \mathrm{G}$ biopsy is sufficient for B3 lesions without atypia, but that lesions identified on $14 \mathrm{G}$ core and those with atypia should continue to have a second-line VAB performed.

\section{P21}

PB.8. Ultrasound-guided excision of fibroadenomas: 9 years' experience in a British breast unit.

P Hamilton, A Sally, CM Lee, A Redman, S Sharma, J Westgarth, A Leaver Gateshead Hospitals NHS Trust, Gateshead, UK.

Breast Cancer Research 2014, 16(Suppl 1):P21

Introduction: Fibroadenomas are benign breast tumours made up of glandular and fibrous tissue. Despite their benign pathology, factors such as size, discomfort, altered breast shape and patient choice sometimes lead to excision. Traditionally, open excision biopsy has been the method of choice for fibroadenoma removal but, since 2005, our breast unit has offered patients minimally invasive ultrasound-guided complete fibroadenoma excision using vacuum-assisted core biopsy (VACB). We present an audit of our practice and patient outcomes regarding VACB excision of fibroadenomas.

Methods: Retrospective audit of all patients that underwent ultrasoundguided VACB excision of fibroadenoma or fibroadenomata (as defined by final excision pathology) in our breast unit between January 2005 and June 2014. The authors obtained data from ultrasound images, radiology reports, pathology records and patient notes. They then performed descriptive statistics.

Results: Eighty-three female symptomatic patients underwent ultrasoundguided VACB fibroadenoma excision as an outpatient. Lesions ranged from 7 to $44 \mathrm{~mm}$ in the longest axis, and from five to 180 cores were taken. Two fibroadenomas recurred $(2.4 \%, 2 / 83)$ : one at 36 months that underwent further VACB excision and one at 10 months that opted for open surgical excision. Three patients developed haematoma. Three patients had incomplete excision noted at the first VACB excision attempt, two of which were completely removed at a second sitting.

Conclusion: Fibroadenoma excision using ultrasound-guided VACB in an outpatient setting is an effective alternative to open excision, and is associated with a low rate of recurrence and morbidity.
P24

PB.23. Breast density in previous screening mammograms of women with and without breast cancer.

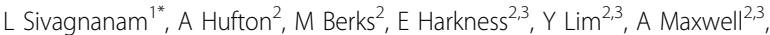
M Wilson ${ }^{3}$, M Bydder $^{3}, \mathrm{~S} \mathrm{Gadde}^{3}$, DG Evans ${ }^{3,4}$, A Howell ${ }^{3,4}$, P Stavrinos ${ }^{3,5}$, S Astley ${ }^{2,4}$

${ }^{1}$ University of Manchester Medical School, Manchester, UK.; ${ }^{2}$ Centre for Imaging Sciences, Institute of Population Health, University of Manchester, UK.; ${ }^{3}$ Nightingale Centre and Genesis Prevention Centre, University Hospital of South Manchester, Manchester, UK.; ${ }^{4}$ Manchester Breast Centre,

Manchester Cancer Research Centre, University of Manchester, Christie Hospital, Manchester, UK.; ${ }^{5}$ The University of Manchester, Manchester Academic Health Science Centre, University Hospital of South Manchester NHS Foundation Trust, Manchester, UK.

Breast Cancer Research 2014, 16(Suppl 1):P24

Introduction: Increased mammographic density is a well-established risk factor for breast cancer; much of the evidence is based on semiautomated or visual assessment of analogue mammograms, although volumetric measures from digitally acquired mammograms are now being reported. It is also possible to quantify volumes of fat and gland from digitised analogue mammograms, given suitable calibration data. We have measured breast density in cancer cases and controls which had previous analogue screening mammograms in the PROCAS (Predicting Risk Of Cancer At Screening) study.

Methods: Forty-nine (44 screen-detected, five interval) cancer cases with film priors were each matched to one control without cancer on the basis of age, BMI, menopausal status and current HRT use. The previous normal screening mammograms for each case were digitised and volumetric breast density measured in the CC view using a calibrated step-wedge imaged alongside the breast. Average area-based percent density was obtained from two readers recording assessments on $10 \mathrm{~cm}$ visual analogue scales (VAS).

Results: Median volumetric percent density was $4.74 \%$ (cancer priors) and $4.77 \%$ (controls); this was not a significant difference $(P=0.436)$. For gland volume the corresponding figures were $37.0 \mathrm{~cm}^{3}$ and $32.1 \mathrm{~cm}^{3}(P=$ $0.667)$, and for VAS percent density were $28.8 \%$ and $27.8 \%(P=0.538)$.

Conclusion: In this sample we did not detect a significant difference between density in prior mammograms of cancer cases and those of controls. The sample size, however, was small. Increasing availability of digital priors of screen-detected cancers will facilitate further exploration of the ability of increased density to predict the development of cancer.

P25

PB.36. Imaging in gynaecomastia: audit of referral and imaging practise to establish referral guidelines.

K Bonam*, A Leaver, S Sharma

Gateshead Hospitals NHS Trust, Gateshead, UK.

Breast Cancer Research 2014, 16(Suppl 1):P25

Introduction: There is variation in the literature and between breast units in use of imaging in clinically suspected gynaecomastia. Our study aim was to audit referral and imaging in our breast unit, formulating guidelines and standardising practise.

Methods: The authors performed retrospective data collection of imaging and pathology for clinically suspected gynaecomastia in our breast unit between January 2012 and May 2014, using hospital radiology and pathology computer information systems. Data included clinical history, clinical score (P1 to P5), ultrasound (U1 to U5) and mammographic scores (M1 to M5). Patients with clinical suspicion of malignancy (written on imaging request or $\mathrm{P} 4 / \mathrm{P} 5$ ) were excluded. Descriptive statistics were performed.

Results: A total of 177 male patients had been referred for imaging with P1 (19), P2 (113), P3 (11) or no P value (34), clinical history stating or querying gynaecomastia. Ultrasound was performed on all patients. All P1 and $\mathrm{P} 2$ patients were $\mathrm{U} 1$ or $\mathrm{U} 2$, and $\mathrm{M} 1$ or $\mathrm{M} 2$ if mammogram was performed (0/19 P1 patients, 20/113 P2 patients, 4/11 P3 patients). In $\mathrm{P} 1$, $P 2$ and no $P$ value patients, $6 / 166$ patients underwent ultrasound-guided biopsy (5/6 B2 gynaecomastia, 1/6 B1 benign angiolipoma). In P3 
patients, two biopsies were performed: B2 gynaecomastia in one patient, but one biopsy (M4U4) revealed B5b invasive carcinoma.

Conclusion: Imaging confirmed the clinical findings in all P1 and P2 patients, but a clinically unsuspected malignancy was diagnosed through imaging of a P3 patient. The findings support guideline formulation where imaging is not indicated for typical (P1, P2) gynaecomastia. Imaging should, however, be performed where there is any doubt at all about the clinical diagnosis.

\section{P27}

PB.10. Stereotactic $20 \mathrm{~mm}$ basket Intact Breast Lesion Excision System biopsy for indeterminate breast microcalcification: pilot study within a UK breast unit.

A Leaver, AJ Potterton, S Athey, CM Lee, S Sharma, A Redman, D Hemming, L Lunt

Queen Elizabeth Hospital NHS Trust, Gateshead, UK.

Breast Cancer Research 2014, 16(Suppl 1):P27

Introduction: For several years our breast unit has evaluated indeterminate breast microcalcification using stereo-guided vacuumassisted core biopsy (VACB) - piecemeal acquisition of unorientated tissue. The Intact Breast Lesion Excision System (Intact) removes a single larger piece of tissue, potentially allowing pathologists to calculate margins and visualise lesion architecture more easily. This study evaluates the role of the $20 \mathrm{~mm}$ Intact in assessment of subcentimetre clusters of indeterminate breast microcalcification.

Methods: The radiology authors performed the Intact biopsy on subcentimetre clusters of indeterminate, sonographically invisible breast microcalcification. A prospective audit was performed, with pathology information from the multidisciplinary meeting. Technical considerations encountered during use of the Intact, sample pathology and short-term patient outcomes are described.

Results: The Intact was performed on 23 female patients, was technically straightforward to operate and was well tolerated by patients. Metal fragments from basket removal were radiologically confused with calcification, but did not result in patient mismanagement.

Pathologists found specimens easier to interpret than VACB samples, although one (1/23) specimen had a $2.5 \mathrm{~mm}$ area of diathermy artefact that could potentially have obscured a lesion. Three (3/23) cases of ductal carcinoma in situ (DCIS) were identified, post-biopsy radiographs consistent with complete excision of microcalcification. The Intact specimen pathology review showed incomplete excision of disease and two of the three cases of DCIS had further DCIS on surgical wide local excision. No invasive disease was identified in any study patient.

Conclusion: In this small pilot study, the Intact has been a reliable and effective tool for stereotactic diagnostic biopsy of breast microcalcification.

PB.28. Determination of recall rates for assessment in women undergoing annual surveillance breast MRI: is a rate $<10 \%$ achievable?. N Healy", S Cosgrove, P Wen, T Kwa, F O'Driscoll, G Offiah, Y Roden, S Sebaoui, K Wolohan, S O'Keeffe

St James Hospital, Dublin, Ireland.

Breast Cancer Research 2014, 16(Suppl 1):P28

Introduction: High recall rates from surveillance breast MRI are associated with patient anxiety and increased workload. NHSBSP guidelines recommend a maximum of a $10 \%$ recall rate with an expected rate of $<7 \%$ in high-risk women undergoing surveillance with breast MRI. Our aims were to review surveillance breast MRIs performed at our institution from 2009 to 2013 to determine the recall rate and cancer detection rates.

Methods: Surveillance MRIs performed in women at high risk of developing breast cancer over a 5 -year period were reviewed. Breast MRI was performed using a standard protocol on a 1.5 Tesla MRI. For all patients with a BIRADS MRI $0,3,4$ or 5 score, additional imaging, modality of biopsy and histology were recorded.

Results: A total of 1,119 surveillance breast MRIs were performed over the 5 -year period. These included women with a known genetic mutation or those at high risk based on genetic assessment. In total, 121 (10.8\%) had BIRADS MRI scores which required recall for further imaging. Seventy-one patients (58.6\%) had a biopsy performed and 19 cancers were detected giving an overall cancer detection rate of $1.7 \%$. Eleven (9\%) were invasive ductal tumours. Of those recalled, $74(61 \%)$ were in the prevalent round of screening.

Conclusion: While our data compare favourably with published data, the breast MRI recall rate is greater than the expected $7 \%$ recommended in the UK. This may be due to large numbers in the prevalent round.

\section{P29}

PB.37. Does conventional imaging accurately predict the extent of disease in women with dense breasts? If so, does this have a significant impact on patient surgical outcome?

$\checkmark$ Scott ${ }^{*}$, S Mohammadi, D Tzias, A Wale, S Heller

St George's Hospital, London, UK

Breast Cancer Research 2014, 16(Suppl 1):P29

Introduction: Breast density is known to mask disease. This study evaluated accuracy of conventional imaging in predicting extent of disease with respect to breast density and surgical outcome.

Methods: Patients treated for biopsy-proven breast cancer between 1 January 2012 and 1 January 2013 were identified. Data regarding patient demographics and lesion characteristics on imaging and on surgical pathology were collected. Mammograms were assigned BI-RADS density scores. Lesion size on imaging was compared with size at final surgical pathology. Effect of breast density on disease underestimation and on final surgical outcome was analysed with two-sided Fisher's exact test.

Results: A total of 237 women were identified; applying exclusion criteria left a final cohort of 165 patients, median age 62 years (range 24 to 96 years). In total, 114/165 (69\%) patients had nondense breasts (BI-RADS 1,2 ) and $51 / 165$ (31\%) had dense breasts (BI-RADS 3, 4).

Lesion size was underestimated on mammography in 131/165 (79.4\%) patients, compared with 138/165 (83.6\%) on ultrasound. Mammography underestimated disease extent in $42 / 51(80.8 \%)$ patients with dense breasts and in $89 / 114(78.1 \%)$ with nondense breasts $(P=0.6776)$ Initially, 97 patients underwent wide local excision, while 68 had mastectomy. Twenty patients needed further surgery; 17 had re-excision and three had completion mastectomy. A total 6/51 (11.8\%) patients with dense breasts required further surgery, compared with $14 / 114(12.2 \%)$ with nondense breasts $(P=1.0)$.

Conclusion: Findings show that conventional imaging underestimates lesion size when compared with final histopathology in dense and nondense breasts. However, this does not have a significant effect on surgical outcome.

\section{P30}

PB.15. An audit of our benign surgical biopsy rate in the prevalent round for the reporting year 1 April 2012 to 31 March 2013.

R Geach", E Kutt, A Valencia

Bristol Royal Infirmary, Bristol, UK

Breast Cancer Research 2014, 16(Suppl 1):P30

Introduction: The NHSBSP has defined standards with regard to benign surgical biopsy rates. In the prevalent population, the minimum defined standard is $<3.6 / 1,000$ with a target of $<1.8 / 1,000$. At the Avon Breast Screening Unit we have been identified as outliers.

Methods: We retrospectively reviewed our benign surgical biopsies from the reporting year 1 April 2012 to 31 March 2013. Thirty-two patients were identified but only 16 were in the prevalent screening population. For each patient the following data were collected: mammographic abnormality; ultrasound abnormality; numerical classification (M1 to M5/U1 to U5 abnormality); lesion size $(\mathrm{mm})$; type of biopsy; B3 histopathological diagnosis; and final histopathological diagnosis.

Results: Forty-four per cent of lesions biopsied and subsequently surgically excised were microcalcifications. Seventy-five per cent of the lesions were $\mathrm{M} 3$ indeterminate and were small, $50 \%$ measuring $<10 \mathrm{~mm}$. All 16 patients had a B3 pathological diagnosis at biopsy. Forty-four per cent were AIDP, $12 \%$ sclerosing adenosis and a radial scar with the remainder other $\mathrm{B} 3$ diagnoses. Of the 11 patients that had AIDP reported in the initial biopsy specimen, foci of atypia were present in just four of these surgical specimens. 
Conclusion: There is inconsistent practice across the UK in the management of B3 lesions. Some may be referred for surgical excision whilst other are removed through vacuum-assisted biopsies. We perform surgical excision for any lesions with atypia. However, to reduce our benign surgical excision rate we aim to increase the number of mammotome excisions for B3 lesions without atypia.

\section{P31}

PB.19. Distortion conspicuity and cancer detection: a comparison of cranio-caudal and mediolateral oblique mammographic projections. S George*, E Muscat, M Sinclair, S Heller

St George's Healthcare NHS Trust, London, UK.

Breast Cancer Research 2014, 16(Suppl 1):P31

Introduction: Mammographic distortions are an indicator of breast malignancy. However, whether distortions and their associated cancers are more readily appreciable on cranio-caudal (CC) or mediolateral oblique (MLO) projection is unexplored. Our study sought to investigate this in view of its potential importance for cancer detection.

Methods: A total of 156 mammograms from the South West London Breast Screening Service were anonymised and randomly ordered. The series included 79 consecutive studies with confirmed distortion and 77 normal studies. Three blinded readers were asked to establish the presence of distortion on separately displayed CC and MLO views, and to score distortion conspicuity ( 1 to 5 ). Readers also reported the degree of confidence (1 to 5 ) that identified distortions represented malignancy.

Results: Distortion detection sensitivity was $75 \%$ for the $\mathrm{CC}$ projection and $64 \%$ for the MLO projection $(P<0.005)$ and specificity was $78 \%$ for the CC projection and $84 \%$ for MLO $(P<0.05)$. Cancer detection sensitivity was $86 \%$ for the CC projection and $75 \%$ for the MLO projection $(P=0.12)$. Positive predictive values were 0.14 and 0.11 respectively. Cancer detection specificity was $55 \%$ for the CC projection and $65 \%$ for MLO $(P<$ $0.05)$. Negative predictive values were 0.98 and 0.97 respectively. In cases of biopsy-proven malignancy, readers were more confident of the presence of cancer on the CC compared with the MLO view $(3.05 \pm 0.28$ vs. $2.22 \pm 0.28 ; P<0.05$ ).

Conclusion: The CC projection was associated with significantly higher distortion detection and higher cancer detection rate than the MLO view. This view was also associated with greater confidence of identifying proven malignancy amongst readers.

P32

PB.20. Audit of 5 years of routine screening mammography of the reconstructed breast.

A Ray", L McNeilly, A Newland, S Flais

Ealing Hospital, Southall, UK.

Breast Cancer Research 2014, 16(Suppl 1):P32

Introduction: In 2008, allowing for an increase in the number of women treated with mastectomy and immediate reconstruction, the decision at our institution was made to carry out mammography of the native and the reconstructed breast at follow-up. A literature review at that time revealed that mammography of the reconstructed breast may help diagnose recurrence before any symptom. This local practice is now audited to evaluate its impact, and whether it should continue.

Methods: All women who had previously undergone mastectomy and reconstruction, and were subsequently imaged with mammography between 2008 and 2013 at Ealing Hospital were included. Subsequent findings and further investigations have been analysed.

Results: Eighty patients treated with mastectomy and reconstruction had regular screening mammograms. Ten local recurrences occurred in six patients. Four recurrences were diagnosed on imaging findings alone (three on mammography, one on MRI). Six recurrences presented with a new symptom (palpable lump or skin abnormality). Routine imaging of the reconstruction generated additional imaging: seven stereobiopsies for microcalcifications (two recurrences) and two ultrasound scans (one recurrence). In the same period, 17 ultrasound scans (five recurrences) and 12 MRIs (one recurrence, but not consistent with the symptom) were requested for clinical concerns.
Conclusion: Routine imaging of the reconstructed breast can diagnose local recurrence with limited harm, generating few additional investigations. However, which patients may benefit and whether this improves outcome is unclear as the number of cases in our audit is small and the event is thankfully rare.

\section{P33}

PB.39. CT or not CT: a systematic review of the breast on CT of the

chest and abdomen over 1 year - another screening tool?.

S Flais *, A Newland, A Flais, B Ellis

Ealing Hospital, Southall, UK

Breast Cancer Research 2014, 16(Suppl 1):P33

Introduction: Images of breast tissue are usually included on CT scans of the chest or abdomen. It is generally thought that CT has a low sensitivity for breast pathology. We tested this assumption to assess the importance of the breast as a review area on CT.

Methods: All CT scans which included some breast tissue and were performed on female patients over a 1-year period at our institution were retrospectively reviewed by a breast radiologist. The breast tissue was scrutinised and, if required, an addendum added to the report and sent urgently to the referring clinician. If the patient was referred for breast assessment, standard imaging findings were compared with the CT appearances.

Results: A total of 2,500 CT scans were reviewed. Thirty per cent of the scans provided imaging of the whole breast. Addendums were added to 112 reports; a recall rate of $5.4 \%$. Sixty-three patients were never referred for breast imaging. The average time from addendum to assessment was 3 months. Forty-nine patients were assessed with breast imaging. Twenty-nine patients underwent a biopsy. Eleven patients had a malignancy. One had a papilloma upgraded to DCIS at surgery.

Conclusion: Significant abnormalities in the breast are visible on CT, and are frequently overlooked. These results have been fed back to our general radiology colleagues and there has been a noticeable increase in reported abnormalities within the breast. We aim to reassess the impact of this work with further audit, which will hopefully demonstrate a reduced rate of missed findings in this area.

P34

PB.29. Retrospective correlation between MRI and histopathology in preoperative assessment of invasive breast carcinoma.

G Gracey* , J McKillen, C Majury, J McAllister

Ulster Hospital, Dundonald, UK.

Breast Cancer Research 2014, 16(Suppl 1):P34

Introduction: Accurate preoperative assessment of a breast tumor has an important role in the planning of breast cancer treatment. The purpose of this study was to assess the accuracy of preoperative MRI in sizing breast tumors of varying pathological subtypes.

Methods: A patient list was populated from the local PACS database. The sample included all patients with a new diagnosis of breast cancer who underwent preoperative contrast-enhanced MRI breast imaging over an 18-month period. The MRI breast images were reviewed and the largest dimension $(\mathrm{mm})$ was recorded. Correlation with the postoperative pathology report was made.

Results: The sample size included 42 patients with 43 breast cancers. Average tumor size was $42 \mathrm{~mm}$. There was direct MRI/pathological size correlation in $41 \%$ of cases. MRI overestimated $19 \%$ of tumor sizes, and underestimated $40 \%$. DCIS was under estimated in $40 \%$. MRI accurately sized $62 \%$ of intermediate DCIS compared with $25 \%$ high-grade DCIS. LCIS was underestimated in $31 \%$. MRI accurately sized $45 \%$ of invasive ductal carcinoma and $40 \%$ of invasive lobular carcinoma.

Conclusion: This study shows that MRI accurately sizes breast cancer tumors in $41 \%$ of cases, overestimates $19 \%$ and underestimates $40 \%$. MRI is more accurate at measuring intermediate DCIS compared with highgrade DCIS. A similar accuracy is found between IDC and ILC. Better preoperative tumor sizing is fundamental for initial surgical planning, decreasing the need for re-excision or mastectomy and therefore appropriate treatment. 
P36

PB.43. A clinical audit of breast cancer staging.

A Sulistijo ${ }^{1 *}$, A Falamarzi ${ }^{1}$, C Chen ${ }^{1}$, C Lightner Ferrer ${ }^{1}$, D Tan ${ }^{1}$, THJ Kong ${ }^{1}$, S O'Keeffe ${ }^{2}$, J Kavanagh ${ }^{2}$

'School of Medicine, Trinity College Dublin, Ireland.; ${ }^{2}$ St James's Hospital, Dublin, Ireland.

Breast Cancer Research 2014, 16(Suppl 1):P36

Introduction: Accurate local and distant staging of breast cancer is essential in determining appropriate disease management and prognosis. Staging investigations delay surgical management and increase cost and manpower requirements. The purpose of the study was to retrospectively assess the utility of imaging in breast cancer staging at St James's Hospital, Dublin between 2012 and 2013.

Methods: The EPR was queried and 559 patients diagnosed with breast cancer between 2012 and 2013 were selected for inclusion in this study. The demographic characteristics, radiological (mammogram, US, MRI, CT), and histopathological records were reviewed. Microsoft Excel 2011 was used to analyse the data using descriptive statistics.

Results: Of 559 patients (age range 19 to 93 years), 298 patients (53\%) underwent staging CT TAP, triggering at least one further investigation in 134 patients (45\%). Forty-three of these staged patients (14\%) had distant metastases; 129 patients (43\%) had normal axillary US, of whom five patients (4\%) had distant metastases. In contrast, $22 \%$ of patients with abnormal axillary US had metastases. Five of 32 patients $(16 \%)$ with triple-negative disease had metastases. Breast MRI was performed in 30\% of patients, triggering further investigations in 37\%. A further 250 investigations were initiated on the basis of CT/MRI in 559 patients.

Conclusion: The excess use of staging CT in patients with early breast cancer and low axillary lymph node burden was not in compliance with the local protocol. Patients with triple-negative disease did not have a higher incidence of metastases at presentation. An average of one further investigation for every two patients is triggered by staging CT and MRI.

P37

PB.21. Is the technical quality of screening mammograms lower in Asian women who cannot understand the English language?.

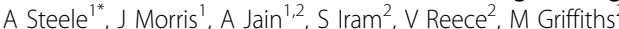

${ }^{1}$ The University of Manchester, UK.; ${ }^{2}$ The Nightingale Centre and Genesis Prevention Centre, Manchester, UK

Breast Cancer Research 2014, 16(Suppl 1):P37

Introduction: Good patient and radiographer communication is vital for good quality mammograms. Many Asian women 47 to 73 years old cannot understand English. The Radiographers QA Guidelines categorise mammographic images as Perfect, Good, Moderate and Inadequate (PGMI). The standards required are 75\% PG, 97\% PGM and $<3 \%$ I (inadequate).

Methods: One hundred women with digital screening mammograms were randomly selected retrospectively and divided into two groups: Group A, 50 Asian women who could not understand English (confirmed from radiographer notes on screening forms); Group B, 50 Caucasian women. Any patients with disability, learning difficulties or previous breast surgery were excluded. Two folders were created on PACS and images were anonymised. The films readers were not aware of the classification of the two groups. All mammograms were independently assessed by two experienced film-reader advanced practitioners using the PGMI scoring system. The final score for each case was reached by consensus with a consultant breast radiologist.

Results: The PGMI mammographic scores for the Asian women are $P(0)$ $G(16), M(26), I(8)$ and scores for the Caucasian women are $P(0), G(31)$, M (18), I (1). The mammographic scores of $32 \%$ PG, $84 \%$ PGM and $16 \%$ I for the Asian women are significantly lower than the scores of $62 \%$ PG, 98\% PGM and 2\% I for the Caucasian women (linear trend test $P=0.001$ ) Conclusion: The mammographic technical quality appears much lower in Asian women who cannot understand English, highlighting a need for improving communication. Further studies are also required to see whether other factors play any role in the poor technical quality in this group.
P38

PB.24. How does volumetric breast density change with time?.

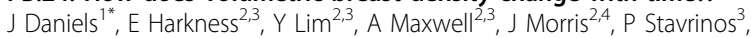
M Wilson ${ }^{3}$, M Bydder ${ }^{3}$, DG Evans ${ }^{3,5}$, A Howell ${ }^{3,5}$

${ }^{1}$ University of Manchester Medical School, Manchester, UK:; ${ }^{2}$ Centre for Imaging Sciences, Institute of Population Health, University of Manchester, UK.; ${ }^{3}$ Nightingale Centre and Genesis Prevention Centre, University Hospital of South Manchester, Manchester, UK.; ${ }^{4}$ University Hospital of South Manchester, Manchester, UK.; ${ }^{5}$ Manchester Breast Centre, Manchester Cancer Research Centre, University of Manchester, Christie Hospital, Manchester, UK. Breast Cancer Research 2014, 16(Suppl 1):P38

Introduction: Breast density is a well-established independent risk factor for breast cancer, but risk may also relate to the rate at which breast density changes over time. Here we aim to establish the baseline rate of change in volumetric breast density in postmenopausal women undergoing mammographic screening.

Methods: Data from 3,620 postmenopausal women attending two consecutive breast screening episodes were obtained from the PROCAS (Predicting Risk Of Cancer At Screening) study database. Women with current/previous breast cancer, current HRT users and those without digital mammographic raw data available were excluded. Volumetric breast density was obtained from digital mammograms taken at two consecutive screening episodes using Volpara software. Mean change in volumetric density was calculated, and its relationship with age, initial density and parity was assessed.

Results: Mean volumetric breast density decreased from $6.43 \%$ at the initial screen to $5.76 \%$ at the subsequent screen, a mean decrease of $0.25 \%$ (percentage points) per year $(P<0.001)$. Fibroglandular volume showed no significant change, whereas breast volume increased by 30.13 $\mathrm{cm}^{3} /$ year $(P<0.001)$, indicating that the decline in density between screens was predominantly due to an increase in nondense breast volume. Decline in breast density was greater with younger age and nulliparity.

Conclusion: Postmenopausal women undergo a mean decrease in volumetric breast density of $0.25 \%$ (percentage points) per year, largely due to an increase in nondense breast volume. Establishing a baseline for volumetric breast density change over time will facilitate further work into whether variation in this pattern provides additional information in predicting breast cancer risk.

P39

PB.30. Apparent diffusion coefficient and fractional anisotropy values as biomarkers for treatment response in breast cancer.

EA Joyce*, AJ Fagan, JP McMorrow, D Byrne, MJ Kennedy, JF Meaney,

SA O'Keeffe

Centre for Advanced Medical Imaging, St James's Hospital, Dublin, Ireland. Breast Cancer Research 2014, 16(Suppl 1):P39

Introduction: The aim was to evaluate whether changes in apparent diffusion coefficient (ADC) and fractional anisotropy (FA) values predict early response in patients receiving neoadjuvant chemotherapy (NACT) for breast cancer and to assess the effect of tumour marker clip placement on ADC values.

Methods: Twenty patients with invasive breast carcinoma underwent MRI at three time points: at baseline (TP0) and following the first (TP1) and second (TP2) cycles of NACT. 3T MRI (Achieva; Philips) was performed using a standard protocol including diffusion-weighted and diffusion tensor imaging. Baseline and sequential data in responder and nonresponder groups were compared. To assess the effects of a commercially available titanium-hydrogel clip on ADC values, DWI was performed on a phantom consisting of a clip embedded in a tumourmimicking target.

Results: At baseline, mean tumour ADC $\left(0.92 \times 10^{-3} \mathrm{~mm}^{2} / \mathrm{second}\right)$ was statistically lower than disease-free fibroglandular breast tissue $\left(1.75 \times 10^{-3}\right.$ $\mathrm{mm}^{2} /$ second $)(P<0.0001)$. Mean FA values of tumour $(\mathrm{FA}=0.139)$ and disease-free tissue $(F A=0.135)$ were similar. Compared with baseline values, tumour ADC of responders significantly increased at TP1 $(P<$ $0.0001)$ and TP2 $(P<0.0001)$ while a significant increase in tumour FA of responders was seen at TP2 $(P<0.008)$. No statistical change occurred in 
tumour $A D C$ or $F A$ values of the nonresponder group. The $A D C$ value of the clip in the phantom $\left(1.7 \times 10^{-3} \mathrm{~mm}^{2} / \mathrm{second}\right)$ was higher than the ADC value of surrounding tumour $\left(1.4 \times 10^{-3} \mathrm{~mm}^{2} / \mathrm{second}\right)$.

Conclusion: Changes in ADC and FA values early in the course of treatment may predict response in patients receiving NACT for breast cancer. The gel-containing clip used for tumour marking prior to NACT results in a source of error when calculating tumour ADC values and should be avoided when drawing regions of interest.

\section{P40}

PB.11. Modern management of acute breast abscesses: radiological interventions replacing surgical incisions?

N Healy, ${ }^{*}$ M Stenson, S Harte

St James Hospital, Dublin, Ireland.

Breast Cancer Research 2014, 16(Suppl 1):P40

Introduction: Traditionally acute breast abscesses were managed with a combination of antibiotics and surgical intervention. Despite advances in imaging techniques and minimally invasive interventions, acute abscesses remain poorly managed with significant treatment delays. The aim of this study was to review management of acute breast abscesses in a symptomatic breast service over 4 years and to develop an updated algorithm for effective and minimally invasive abscess management.

Methods: From January 2010 to June 2014 all acute abscesses referred to the radiology service were retrospectively reviewed with attention to patient demographics, US findings, aetiology, radiological or surgical intervention, treatment duration and outcome. Total number of ultrasounds (USs), aspirations and US-guided catheter placements were recorded.

Results: A total of 203 acute abscesses attended with 160 US-guided aspirations and 43 US-guided catheter placements over 4 years. Patients required on average 2.3 US and 1.4 aspirations during each acute episode. Puerperal abscesses accounted for 38 (23\%) of the aspiration cases and for $29(67 \%)$ of the catheter cases. The mean abscess size managed with drainage catheters and aspiration was $4.4 \mathrm{~cm}$ and $2.7 \mathrm{~cm}$ respectively. Four (2.2\%) patients ultimately required surgical intervention.

Conclusions: Radiological assessment and minimally invasive intervention is an accessible and effective strategy in the management of acute breast abscesses. This approach limits more aggressive surgical interventions with improved patient acceptability. An updated treatment algorithm should be adopted in all symptomatic breast clinics to ensure timely treatment and optimise outcome.

P41

PB.31. Occult breast carcinoma: an experience at a single unit.

S Merai", C Low, V Gaur, S Muthyala

UHCW NHS Trust, Coventry, UK.

Breast Cancer Research 2014, 16(Suppl 1):P41

Introduction: The breast carcinoma presents rarely as metastases to the axillary nodes without an obvious breast lesion seen on conventional imaging such as mammography and US. In this study we looked at the usefulness of DCE-MRI of the breast in identifying these tumours at a single institute. In addition we evaluated the use of second-look US and MRIguided biopsy.

Methods: A total of 10 patients were identified from our local MR database who were diagnosed with occult breast cancer between February 2012 and April 2014. The data were collected from PACS and the hospital RIS.

Results: All patients presented with axilllary nodes and had a normal clinical examination of the breast and had normal mammograms. The majority of these patients (80\%) had mixed-density breast parenchyma. In $80 \%$ of patients the cancer could be diagnosed on the DCE-MRI of the breast. The remaining two patients had normal MR examination. The morphological features of the lesions were nonmass-like enhancement, spiculate and lobulated masses, ranging in size from 6 to $35 \mathrm{~mm}$. Fifty per cent of these patients demonstrated type 3 kinetic curves. In $62.5 \%$ of patients with positive MR, the lesion could be identified and biopsied on the second-look US. In the remaining two cases the tumour was diagnosed with MRI-guided biopsy. Most patients underwent wire-guided WLE and ANC. The majority of these cancers (75\%) were ductal NST.
Conclusion: Our experience is similar to previously published literature in that MRI aids in identifying most of the occult cancers.

\section{P42}

PB.3. Detection of lymph node metastases in newly diagnosed breast cancer patients with axillary ultrasonography in combination with the Memorial Sloan Kettering Cancer Centre normogram as a prediction tool. CS Low*, S Meraj, M Sreenivas

University Hospitals Coventry and Warwickshire NHS Trust, Coventry, UK. Breast Cancer Research 2014, 16(Suppl 1):P42

Introduction: Current axillary lymph node assessment criteria by ultrasonography (US) depends on cortical thickness $>3 \mathrm{~mm}$, focal eccentric cortical thickness and lobulated cortex. Combination with a prediction tool may define better patient management strategies.

Methods: Newly diagnosed breast cancer patients within a 1-year period were randomly selected retrospectively. Inclusion criteria include patients with final histological correlation following resection. The Memorial Sloan Kettering Cancer Centre breast cancer normogram has been utilised. Axillary nodal US findings and histological correlation from fine needle aspiration cytology (FNAC)/core biopsies were obtained.

Results: A total of 70/160 randomly selected patients fulfilled the inclusion criteria.

In total, 28/70 patients were positive for axillary nodal metastases. A total $15 / 28$ patients underwent $\mathrm{FNAC/core} \mathrm{biopsy} \mathrm{and} 11$ were positive. In the remaining 13 patients, nodal metastases were detected by sentinel lymph node biopsy (SLNB) in 10 patients and initial axillary node clearance (ANC) in three patients. These 13 patients had a probability range of 26 to $91 \%$ (mean 59\%). The overall probability range in all 28 patients is 6 to $97 \%$ (mean 64\%). Forty-two patients did not have lymph node metastases. One patient did not have axillary US assessment. In total, 10/41 patients had abnormal nodes on US that were negative on FNAC/core biopsy. All 42 patients had surgical lymph node assessment (38 had SLNB, four had ANC). The overall probability range is 9 to $80 \%$ (mean $36 \%$ ).

Conclusion: The detection of axillary nodal metastases with US remains low but combination with the normogram prediction tool may be helpful to determine patients with high probability to have repeated US assessment and sampling of normal-looking nodes to increase the detection rate.

P43

PB.4. Ultrasound false negative preoperative axillary assessment in breast cancer patients undergoing sentinel node biopsy.

L Grosvenor*, M Al-Attar, D Lister, G McDonald, S Kaneri, N Hartley, M Hoosein, L Sundaram, E Denton

Breast Care Centre, Glenfield Hospital, UHL, Leicester, UK. Breast Cancer Research 2014, 16(Suppl 1):P43

Introduction: Concern was raised by our local pathology unit regarding an increase in the number of positive sentinel node biopsies (SNB). As an imaging group we did not have the evidence base to refute the concerns raised and therefore undertook a 6-month retrospective review of unit performance.

Methods: Pathology provided a database list of all patients they regarded as positive SNB (false negative US assessment). The period covered was 6 months (1 November 2012 to 30 April 2013). This was referenced against all axillary surgeries during the same period.

Results: The pathology database extended to 98 patients. Multiple duplications were identified and correctly identified preoperative $C 5$ axillae were excluded. A final cohort of 43 positive SNB (false negative US) assessments were identified from the 266 SNB procedures performed in the time period. The 13 operators included locums, trainees, multiprofessionals and external consultant staff. All operators had at least one false negative assessment of the axilla. Positive nodal histology was reported for 14 cases as micro-metastases or isolated tumour cells (ITC) and in 29 cases as macro-metastases/extranodal spread or multiple involved nodes. Eleven per cent of SNBs were considered false negative US assessments (29/266). Our NPV calculates at $83.8 \%$ (43 patients $=223$ / 266) for all surgical positive axillae and $89 \%$ with exclusion of micrometastases/ITC. 
Conclusion: No individual performance or unit concern was outside the published literature ranges. Individual clinical cases of concern were identified for peer review/clinical governance. Imaging review has led to consolidation of the working practice and an ongoing prospective audit with continual review. However, the balance between adequate assessment, capacity and resource needs defining. How good is good enough?

\section{P44}

PB.40. What happens to the ductal carcinoma in situ in HER2-positive cancers treated with neoadjuvant chemotherapy and trastuzumab?. R Millican-Slater, D Dodwell, K Horgan, M Mcmahon, B Dall, N Sharma Leeds Teaching Hospital NHS Trust, Leeds, UK.

Breast Cancer Research 2014, 16(Suppl 1):P44

Introduction: There are few data regarding the effect of neoadjuvant chemotherapy (NACT) and trastuzumab on any ductal carcinoma in situ (DCIS) associated with the HER2-positive invasive carcinoma. HER2positive breast cancers are more likely to achieve a pathological complete response (defined as absence of invasive tumour in the final excision specimen allowing for the presence of DCIS). We review our data to see what happens to HER2-positive DCIS associated with HER2-positive invasive cancer treated with NACT.

Methods: All cases that were HER2-positive from our local NACT database were identified from 2010 to 2012. The imaging features, core biopsy and final histology were documented.

Results: A total 41 of 150 patients that received NACT (anthracycline and taxane based) were HER2-positive and treated with trastuzumab. In total, 40/41 cases had surgery following neoadjuvant treatment, 24/41 (59\%) cases had calcifications on mammography, and 21/41 (51\%) obtained a pCR. Of the 24 cases with calcification, 14 (44\%) had a pCR with 9/14 (29\%) having residual DCIS. Seven of 24 cases had a WLE in which the calcifications on mammography were unchanged in extent or appearance. A total $17 / 41$ were without calcification, seven (57\%) had a pCR with $2 / 7$ $(62 \%)$ having residual DCIS that was noncalcified.

Conclusion: $\mathrm{pCR}$ is higher in the HER2-positive group compared with the HER2-negative group (51\% vs. $20 \%$ ). In total, $48 \%$ achieved a pCR with no residual DCIS but $52 \%$ still had residual DCIS, suggesting that trastuzumab may be less effective in treating HER2-positive DCIS.

P45

PB.17. Are patients who have had total body irradiation at similar risk of breast cancer to those having mantle radiotherapy? A review of the evidence and suggestions on breast imaging surveillance.

J Muscat ${ }^{1,2^{*}}, \mathrm{G}$ Rubin

${ }^{1}$ Brighton and Sussex University Hospitals NHS Trust, Brighton, UK.; ${ }^{2}$ Mater

Dei Hospital, Msida, Malta.

Breast Cancer Research 2014, 16(Suppl 1):P45

Introduction: There are an increasing number of total body irradiation (TBI) survivors. UK haematology and oncology units follow international guidelines and advise that they should have annual mammography from age 40 .

Methods: A literature review found a single large study published in Blood in 2008 based on 3,337 women from 83 centres, who had survived at least 5 years after transplant [1]. The radiation dose was 8 to 16 Grey distributed homogeneously rather than to the mediastinum in mantle radiotherapy. Fifty-two women developed breast cancer at a median of 12.5 years after transplantation, and 47 of these had TBI as well as bone marrow transplant. Their breast cancer incidence was compared with the standardised incidence.

Results: The study found that: overall breast cancer risk was moderately increased, by 2.2 ( $95 \% \mathrm{Cl}=1.7$ to 2.9 ); risk was concentrated in younger patients, those treated in their teens and twenties; and the standardised incidence of those presenting after 20 years was also 10 -fold that expected.

Conclusion: There are similarities between the risks of TBI and mantle radiotherapy, cancers occurring if irradiated when young and cumulative risk increasing disproportionately with length of follow up. More work needs to be done comparing the radiation dose to the breast and relative breast cancer risk of TBI and mantle radiotherapy. If this shows that the dose and risk are similar, then TBI patients are most likely to benefit from similar breast imaging follow up.

Reference

1. Freidman DL, Rovo A, et al: Increased risk of breast cancer among survivors of allogenic hemopoetic cell transplantation. Blood 2008, 11 P939-P944.

P46

PB.25. Implementation of two new digital mammography technologies for Breast Test Wales: more or less accurate?.

T Evans*, B Burlton, K Gower-Thomas

Royal Glamorgan Hospital, South Wales, UK.

Breast Cancer Research 2014, 16(Suppl 1):P46

Introduction: The aim of this study is to compare the performance of Sectra and Hologic digital mammography, two different technologies introduced to Breast Test Wales in 2012 for breast cancer screening. Specifically, any variation in findings and whether different types of cancer - for example, invasive or non-invasive - are better identified by either system.

Methods: This is a retrospective study of 50,000 consecutive mammograms starting from 2012 of females aged 50 to 70 carried out in the Breast Test Wales mobile screening units. All cancers identified in the two arms of the study were detailed and compared specifically with regards to type (ductal or lobular), size, grade, invasion and node status. Performance of each mammography technology with regard to the detection rate of each cancer type was analysed for any statistically significant difference.

Results: Of 50,000 women screened, 500 cancers were detected with no significant difference in overall invasive cancer detection, invasive ductal or invasive lobular detection found between the two screening technologies. However, Hologic $(1.44$ per 1,000$)$ was found to have a significantly higher non-invasive cancer detection rate than Sectra $(2.88$ per 1,000$), P<0.001$.

Conclusion: While Hologic and Sectra have been found to be comparable in terms of invasive cancer detection, this study shows a difference in the number of non-invasive cancers they detect. Further study and a decision as to whether the difference is clinically significant long term are now required.

P47

PB.16. Breast screening mammograms: recall or not to recall. What is the golden ratio?.

AR Sever ${ }^{1,2^{*}}$, R Pietrosanu ${ }^{2}$, J Weeks ${ }^{2}$, P Mills ${ }^{2}$

${ }^{1}$ University of Hacettepe, Ankara, Turkey.; ${ }^{2}$ Maidstone Hospital, Maidstone, UK. Breast Cancer Research 2014, 16(Suppl 1):P47

Introduction: The accuracy of prospectively categorised screening mammograms prior to assessment was evaluated and the individual recall and cancer detection rates for each category were studied.

Methods: The screening mammograms of women who attended the National Health Service Breast Screening Program over a period of 5 years (April 2008-March 2013) were included in the study. The recalled patients' films were prospectively categorised into one of six groups: patients with normal mammograms requiring clinical recall; probably benign; probably benign/suspicious; suspicious; suspicious/malignant; and malignant. Recalled patients were subsequently assessed with additional mammographic views, \pm ultrasound and/or needle biopsy. The recall and cancer detection rates of each category were individually calculated.

Results: A total of 86,405 mammograms $(17,881$ prevalent and 68,524 incident rounds) were read and 4,125 patients were recalled for assessment (overall recall rate $=4.8 \%$ ). A total of 721 patients were diagnosed with cancer $(0.8 \%)$. The prevalent recall rate was $9.1 \%$ (recommended programme target $<7 \%$ ) and incident recall rate was $3.6 \%$ (target $<5 \%$ ).

Conclusion: Mammograms showing minimal signs of cancer that were categorised as 'probably benign' comprised the largest recall group with a relatively low detection rate $(7 \%)$. If patient recall was limited to only more suspicious groups (that is, $\mathrm{PB} /$ suspicious and above), the overall recall rate would have dropped to $1 \%$ from $4.8 \%$; however, $28 \%$ of the cancers would have missed. Patient anxiety generated due to false positive recalls and 
the cost of assessment clinics needs to be carefully balanced against a higher cancer detection rate.

P48

PB.5. Accuracy of axillary nodal staging on MRI of the breasts: correlation with ultrasound of the axilla and histopathology findings. M Wilson ${ }^{1 *}$, A Maxwell ${ }^{1,2}$, S Gadde ${ }^{1}$, E Hurley ${ }^{1}$, M Bydder ${ }^{1}$, E Harkness ${ }^{1,2}$, M Ewins ${ }^{3}$, Astley $^{1,2}, Y$ Lim $^{1,2}$

${ }^{1}$ The Nightingale Centre and Genesis Prevention Centre, University Hospital of South Manchester, Manchester, UK.; ${ }^{2}$ Centre for Imaging Sciences, University of Manchester, UK:; ${ }^{3}$ University of Manchester Medical School, Manchester, UK.

Breast Cancer Research 2014, 16(Suppl 1):P48

Introduction: Ultrasound (US) is routinely used for preoperative staging of the axilla in women with breast cancer but this has limitations with variable sensitivities published in the literature. Magnetic resonance imaging (MRI) of the breasts is frequently performed for preoperative staging of the breasts, and the axilla can be visualised on these scans. The aim of this study is to assess the accuracy of axillary nodal staging on MRI of the breasts.

Methods: A 4-year retrospective study was performed that included 205 women with breast carcinoma who underwent preoperative staging MRI of the breasts. Two consultant radiologists reviewed the MRI. The nodes were assessed for loss of fatty hilum, eccentric cortical thickness and maximal short axis diameter $>10 \mathrm{~mm}$. The findings were recorded on a proforma and subsequently correlated with the US findings and surgical histology results.

Results: For the 205 cases, the sensitivity $(95 \% \mathrm{Cl}$ ) was $55 \%$ (48 to $62 \%$ ) for MRI and $49 \%$ (42 to $56 \%$ ) for US. The specificity was $88 \%$ ( 84 to $92 \%$ ) for MRI and $98 \%$ (96 to 100\%) for US. The combination of MRI and US resulted in an increase in sensitivity to $79 \%$ (73 to $85 \%$ ) with a specificity of $84 \%$ ( 79 to $89 \%$ ).

Conclusion: There was no statistically significant difference in the sensitivity and specificity of preoperative axillary nodal staging between MRI and US. The combination of MRI and US has been shown to have significantly higher sensitivity. Careful evaluation of the axilla on MRI to identify the cases for second-look US may increase the accuracy of preoperative staging of the axilla.

\section{P49}

PB.44. Do qualitative patterns of stiffness help differentiate benign from malignant breast masses of similar stiffness during shear wave elastography?.

M Elseedawy ${ }^{1 *}$, P Whelehan ${ }^{2,3}$, S Vinnicombe ${ }^{2,3}$, D McLean ${ }^{3}$, K Thomson $^{3}$, A Evans ${ }^{2,3}$

${ }^{1}$ University of Dundee, UK.; ${ }^{2}$ Dundee Cancer Centre, Dundee, UK.; ${ }^{3}$ Ninewells Hospital, Dundee, UK.

Breast Cancer Research 2014, 16(Suppl 1):P49

Introduction: Previous studies assessing qualitative patterns of stiffness in breast lesions have not matched benign and malignant lesions for quantitative stiffness. The purpose of this study is to compare the qualitative patterns of stiffness of benign and malignant lesions matched for quantitative stiffness.

Methods: A total of 158 consecutive histologically confirmed benign lesions with a mean stiffness $>30 \mathrm{kPa}$ were identified from a prospective database. Forty-nine cancers with the same distribution of stiffness as the benign lesions were identified for comparison. The following features were assessed by two observers; BIRADS score, whether there was stiffness in the lesion, outside the lesion, Tozaki classification, homogeneity of stiffness, the presence of a ring sign, stiffness adjacent to the skin or muscle, and the assessed likelihood of the stiffness being artefactual. Statistical analysis was carried out using the chi-square test.

Results: Benign and malignant lesions both had a mean stiffness of $73 \mathrm{kPa}$. The following features show a significant association with malignancy; BIRADS classification 4 or 5 (45 (91\%) vs. $99(63 \%), P<0.0001)$, Tozaki classification 3 and $4(43(88 \%)$ vs. $85(54 \%), P<0.001)$, and presence of a ring sign $(40(82 \%)$ vs. $78(49 \%), P<0.001)$. The following features were more common in benign lesions; stiffness in the lesion $(103(65 \%)$ vs. 17 $(35 \%), P=0.0002)$, stiffness adjacent to skin $(99(63 \%)$ vs. $19(39 \%), P=0.003)$ and stiffness thought to be probably artefact (74 (47\%) vs. $5(10 \%)$ $P<0.0001$ ).

Conclusion: Qualitative features show significant differences in frequency when comparing benign and malignant masses. Qualitative features could be used to help classify breast lesions during SWE.

P50

PB.9. Randomised controlled trial of stereotactic $11 \mathrm{G}$ vacuum-assisted core biopsy for diagnosis and management of malignant microcalcification.

S Bundred ${ }^{1 *}$, A Maxwell $^{1}$, J Morris ${ }^{1,2}$, J Harake $^{3}$, S Whiteside $^{1,2}$, J Zhou $^{1}$, $N$ Bundred $^{1,2}$

${ }^{1}$ University Hospital South Manchester, Manchester, UK.; ${ }^{2}$ University of Manchester, UK.; ${ }^{3}$ Royal Bolton NHS Foundation Trust, Bolton, UK. Breast Cancer Research 2014, 16(Suppl 1):P50

Introduction: Vacuum-assisted biopsy (VAB) may improve accuracy of diagnosis of malignant microcalcification (MM) compared with $14 \mathrm{G}$ core biopsy (SCNB), thus reducing requirements for further surgical procedures.

Methods: VAB and SCNB were compared in a randomised controlled trial (1:1) in first-line diagnosis of MM and subsequent surgical outcomes in two UK breast screening units. Participants gave written informed consent prior to randomisation. Exclusions included bleeding diathesis or comorbidity precluding surgery. SCNB was performed by advanced radiography practitioners and VAB by radiologists. Quality-of-life $(\mathrm{QOL})$ was assessed using the EORTC QLQ BR-23 questionnaire pre biopsy, 2, 6 and 12 months post randomisation. Final pathological diagnosis was compared with the initial biopsy result. A total of 110 participants were required to show a $25 \%$ improvement in diagnosis with VAB compared with SCNB (90\% power).

Results: PPV for MM biopsy was $30.2 \%$. Groups were evenly matched for age and lesion size $(75 \%<15 \mathrm{~mm})$. Eligibility was assessed for 787 cases; 129 women were randomised. Diagnostic accuracy of VAB was $86 \%$ and SCNB was $84 \%$, which precluded any effect on surgical outcomes; $12.5 \%$ of VAB cases (8.7\% SCNB) upgraded from DCIS at surgery. Following VAB, 44\% cases required repeat surgery $(29 \% \mathrm{SCNB})$. VAB took longer $(P<0.001)$ and yielded more specimens $(12$ vs. 8$)(P<0.01)$. Three VAB $(4.7 \%)$ procedures were complicated by severe bleeding. Significant falls in QOL scores for global health $(P<0.001)$ and social functioning $(P<0.04)$ were observed over time for both groups.

Conclusion: SCNB and VAB were equally accurate in the first-line diagnosis of malignant microcalcification.

P51

PB.45. Correlation of perilesional tissue stiffness measured by ultrasound strain elastography with breast density.

A Di Batista ${ }^{1 *}$, R English², R Sidebottom², R Adams², L Winter ${ }^{2}$, A Noble $^{1}$, A Harris'

'Oxford University, Oxford, UK.; ${ }^{2}$ Oxford University Hospitals NHS Trust, Oxford, UK.

Breast Cancer Research 2014, 16(Suppl 1):P51

Introduction: Over an 11-month period a series of patients with screendetected breast masses were recruited with ethical approval into a BRCfunded study designed to evaluate the use of ultrasound elastography in the sizing of early breast cancer. We present results and imaging examples from 71 patients in whom BIRADS evaluation of breast density is correlated with elastography findings of the perilesional tissue.

Methods: Ultrasound elastography was performed in addition to the standard assessment modalities using a Zonare z.one US unit. Elastography data were post processed and were classified in terms of the strain characteristics of the perilesional tissue into stiff or not stiff. Standard mammogram projections were evaluated by two experienced breast radiologists (RE and RA) for breast density using the BIRADS system of classification. Intraobserver (RE) and interobserver (RE, RA) consistency was evaluated. Histological diagnosis subsequently demonstrated invasive disease in 55 patients and noninvasive in 16 patients.

Results: Intraobserver and interobserver consistency was $83 \%$ and $82 \%$ respectively. There was no case exceeding one category of difference. No correlation was demonstrated between perilesional tissue characteristics and background breast density. Sixty-two cases were classified as BIRADS 1 
and 2, of whom 38 had perilesional stroma measures as stiff and 24 as not stiff. Nine cases were classified as BIRADS 3 or 4 , of whom five had perilesional stroma measures as stiff and four as not stiff.

Conclusion: These results suggest that perilesional tissue stiffness is not closely related to the underlying breast density.

P54

PB.32. Type of breast surgery in patients undergoing neoadjuvant chemotherapy: role of DCE-MRI.

M Halim*, M Sreenivas

UHCW NHS Trust, Coventry, UK.

Breast Cancer Research 2014, 16(Suppl 1):P54

Introduction: The aim was to assess the role of MRI in neoadjuvant chemotherapy and whether this has helped the surgical decision. A secondary aim was to identify the pattern of malignant lesions seen on MRI and to correlate this with the surgical decision.

Methods: Twenty-seven consecutive patients who had MRI scans prior to neoadjuvant chemotherapy were identified. Nineteen patients had a postchemotherapy scan.

The pattern of malignant lesions was identified on the baseline MRI and divided into five previously described categories (circumscribed (type 1), nodular (type 2), diffuse (type 3), patchy enhancement (type 4) and septal spread (type 5)).

Results: Out of the 27 patients, 11 patients had mastectomies and 16 underwent breast-conservation surgery (BCS). From the 19 patients who had a postchemotherapy MRI, four had complete therapeutic response, 14 had partial response and one showed disease progression. Of the 27 patients, 14 had circumscribed malignancy with an average size of $39 \mathrm{~mm}$ (range $=25$ to $60 \mathrm{~mm}$ ), two had a nodular pattern, four were diffuse, six showed patchy enhancement and one showed septal spread. Of the 14 patients with circumscribed malignancy, 13 had BCS and one had mastectomy. All four patients with diffuse malignancy had mastectomies. Five patients with types 2, 4 and 5 (nine patients in total) MRI patterns had mastectomies.

Conclusion: MRI was helpful in the surgical decision where it showed either complete or partial therapeutic response in circumscribed malignancies, thereby avoiding unnecessary mastectomies. In diffuse malignancies, the decision was invariably mastectomy irrespective of the response to neoadjuvant chemotherapy.
P55

PB.38. Extent of agreement between radiological and pathological size and factors affecting completeness of excision in breast-conserving surgery for invasive breast cancer.

C Newlands*, M Dixon, L Williams

University of Edinburgh/Edinburgh Breast Unit, Edinburgh, UK.

Breast Cancer Research 2014, 16(Suppl 1):P55

Introduction: Completeness of excision is the most important factor influencing local recurrence for invasive breast cancer. The aim of this study was to determine whether or not radiological underestimation of tumour size increased the risk of incomplete excision in patients undergoing breast-conserving surgery (BCS).

Methods: This was a retrospective study of 311 women diagnosed with invasive breast cancer and treated with BCS. Data were extracted from patient notes.

Results: Mean underestimation of tumour size was $11.69 \mathrm{~mm}$ for mammography and $17.18 \mathrm{~mm}$ for ultrasonography in the incomplete excision group. For every $1 \mathrm{~mm}$ increase in mammographical and ultrasonographical underestimation, the risk of incomplete excision rose by $10 \%$ and $14 \%$ respectively. T stage, maximum tumour diameter, multifocal disease, an in situ component and mammographical underestimation all significantly increased the risk of incomplete excision when applied to a best-fit model. The larger the size of the in situ component, the greater the risk of both radiological underestimation and incomplete excision.

Conclusion: Underestimation of tumour size by current radiological techniques increases the risk of incomplete excision in women undergoing BCS. Better preoperative assessment of tumour size is required to reduce this risk. Larger, prospective studies are needed to verify the associations found here.

Cite abstracts in this supplement using the relevant abstract number, e.g.: Newlands et al:: PB.38. Extent of agreement between radiological and pathological size and factors affecting completeness of excision in breast-conserving surgery for invasive breast cancer. Breast Cancer Research 2014, 16(Suppl 1):P55 